\title{
Treatment Resistant Hypertension: A Pragmatic Management Approach
}

\author{
Brent M Egan
}

\section{ABSTRACT}

Treatment resistant hypertension ( $\mathrm{TRH}$ ) is defined by office blood pressure (BP) uncontrolled on $\geq 3$ or controlled on $\geq 4$ antihypertensive medications, preferably at optimal doses and including a diuretic. Among treated hypertensives, $~ 30 \%$ of uncontrolled and $10 \%$ of controlled individuals have apparent treatment resistant hypertension (aTRH). Apparent treatment resistant hypertension is used when optimal therapy, patient adherence, and BP measurement artifacts are unknown. In $\geq 50 \%$ of aTRH patients, BP measurement artifacts ('office' TRH), suboptimal regimens, or suboptimal adherence are present, i.e. pseudoresistance. Patients with 'office' TRH have fewer cardiovascular events (CVE) than those with persistent hypertension. Patients with suboptimal regimens or adherence and persistent hypertension appear to have excess CVE. 'Office' TRH is minimized by averaging several BP values obtained with an accurate, automated monitor, while the patient is alone in the examination room'. Home or ambulatory BP monitoring directly confirm 'office' TRH or persistent hypertension. Optimal therapy is reasonably defined by $\geq 3$ different antihypertensive medication classes, e.g. thiazide-type diuretic, renin-angiotensin blocker and calcium antagonist at $\geq 50 \%$ of maximum recommended doses. Intensifying diuretic therapy is effective for controlling many TRH patients who are volume expanded. Personalized strategies, e.g. renin or hemodynamics, can inform successful therapy. Patient blood pressure self-monitoring and attention to adverse effects, medication costs, and pill burden can improve adherence. Suspected secondary hypertension should be evaluated and interfering substances or medications discontinued. These approaches will identify or correct the problem in $\sim 80 \%$ of aTRH patients. Referral to a hypertension specialist is recommended for TRH patients not successfully managed with the pragmatic approach outlined.

Keywords: Cardiovascular disease, Nonadherence, Office resistance, Pseudoresistant hypertension, Spironolactone, Treatment resistant hypertension.

How to cite this article: Egan BM. Treatment Resistant Hypertension: A Pragmatic Management Approach. Hypertens J 2015;1(1):106-110.

Source of support: Nil

Conflict of interest: None

\section{Medical Director}

Care Coordination Institute, Greenville Health System; Department of Medicine, University of South Carolina, School of Medicine, Greenville, South Carolina, United States

Corresponding Author: Brent M Egan, Medical Director Care Coordination Institute, 300 East McBee Avenue Greenville, South Carolina, United States, Phone: 864-522-2261 e-mail: began@ccihealth.org

\section{INTRODUCTION}

Treatment resistant hypertension (TRH) was defined as blood pressure (BP) uncontrolled on $\geq 3$ or controlled on $\geq 4$ BP medications prescribed at optimal doses and preferably including a diuretic. ${ }^{1}$ The term apparent aTRH is frequently used, since measurement artifacts, suboptimal treatment regimens, i.e. contributors to pseudoresistance, are often unknown. ${ }^{2}$ Prevalent apparent treatment resistant hypertension (aTRH) among treated uncontrolled hypertensives nearly doubled from 16 to $28 \%$ between 1988-1994 and 2007-2010. Simultaneously, the proportion of adults with controlled hypertension rose from 27 to $52 \%$. More intensive treatment accounted for the increase in hypertension control together with factors predisposing to TRH including increases in age, obesity, diabetes and kidney disease. ${ }^{1,2}$ The predisposing factors are all associated with insulin resistance, which is more common in adults with TRH. ${ }^{3}$

Apparent TRH impacts roughly $11 \%$ of all hypertensive adults in the US, which includes individuals who are unaware of their hypertensive and aware individuals who are untreated. Among hypertensive adults on treatment, $\sim 30 \%$ of treated uncontrolled and $10 \%$ of treated controlled adults in the US or $\sim 8$ million individuals. ${ }^{2,4}$ Given the high prevalence of aTRH and unfavorable prognosis, ${ }^{1,2,4}$ it is important for primary care clinicians to identify and manage most of these individuals as referring all for specialty care is impractical. This review outlines a pragmatic approach to evaluating and treating aTRH that can be implemented in many primary care settings (Table 1). ${ }^{1}$

Measurement artifacts are common in uncontrolled aTRH. ${ }^{5-7}$ Since measurement artifacts are very common and affect 30 to $50 \%$ of aTRH patients, a good starting point for evaluation is an accurate and representative BP. In other words, a BP can be measured accurately in the office setting by trained individuals carefully following standardized measurement protocol. However, that carefully measured BP is not necessarily representative of usual daytime readings. Another compelling reason to obtain a BP value more representative of usual daytime values is that 'office' TRH has a better prognosis than true TRH. ${ }^{6,7}$ Patients trained in BP self-monitoring who 
Table 1: A seven-step plan for evaluation and management of treatment resistant hypertension ${ }^{1}$

1. Confirm treatment resistance: Office blood pressure $>140 / 90$ on $\geq 3$ antihypertensive medications at optimal doses and preferably including a diuretic.

2. Exclude pseudoresistance: Is the patient adherent with an optimal treatment regimen? Is out-of-office blood pressure elevated?

3. Identify and address contributing lifestyle factors, e.g. obesity, physical inactivity, excess alcohol and high salt intake

4. Discontinue or reduce interfering substances, e.g. NSAIDs, sympathomimetics, oral contraceptives, erythropoietin, ephedra (nonprescription weight loss)

5. Screen for secondary causes of hypertension, e.g. obstructive sleep apnea, primary aldosteronism, chronic kidney disease, renal artery stenosis, pheochromocytoma

6. Continue to optimize pharmacologic treatment: Enhance diuretic therapy (see text); consider alpha1 and beta1 adrenoceptor blockade in patient with an otherwise optimal regimen (avoid combining beta-blockade and nondihydropyridine CCB)

7. Refer to a hypertension specialist for definitive evaluation and treatment of known or suspected secondary cause(s) of hypertension or if blood pressure remains uncontrolled after 6 months

use an accurate device can provide reliable out-of-office readings. Using an accurate, validated ambulatory BP monitor is another method for obtaining useful BP values outside the office setting. ${ }^{8}$ Self-monitored BP and ambulatory BP monitoring both provide prognostically important information beyond that of office BP. ${ }^{9}$ With either of these techniques, a nonhypertensive daytime BP is $<135 /<85 \mathrm{~mm} \mathrm{Hg}$. Using an accurate, automated office $\mathrm{BP}$ monitor to obtain a series of readings with the patient alone in the examination room minimizes the office effect and better approximates usual daytime readings. ${ }^{10}$ The Canadian Hypertension Guidelines recommend using a $B P$ value of $<135 /<85$ as nonhypertensive when the mean of several automated readings is obtained in the office setting as described above. ${ }^{11}$

\section{Suboptimal Treatment Regimens}

In working with several hundred primary care practice settings, we documented that $\sim 50 \%$ of patients with uncontrolled aTRH were not prescribed optimal therapy defined as three different antihypertensive medications including a diuretic at $\geq 50 \%$ of the maximum recommended or approved doses, e.g. hydrochlorothiazide $25 \mathrm{mg}$, lisinopril $20 \mathrm{mg}$, and amlodipine $5 \mathrm{mg}$ daily. ${ }^{3}$ While some patients may not tolerate optimal therapy, clinicians have many opportunities to improve BP control by optimizing prescribed antihypertensive medications in their patients with aTRH who have hypertension outside the office setting.
Inadequate adherence or failing to take $\geq 75 \%$ of prescribed medication, impacts $\sim 10$ to $60 \%$ of aTRH. ${ }^{2,3}$ Direct questions on medication adherence often elicit unreliable responses. Inviting patients to share concerns about costs, side effects or other barriers can be useful in obtaining clinically useful information. Any admission of nonadherence is often associated with taking $<75 \%$ of prescribed medication. Concerned and caring family members often times have insight on patient adherence and barriers.

Medication possession ratio (MPR) or the percentage (fraction) of days the patient possesses the medication during a given time period, e.g. 6 to 12 months, is a reasonable proxy for adherence. ${ }^{7}$ A conversation with the pharmacist and examining pill bottles to assess the number of prescription refills relative to the dates of the initial prescription and visit are useful, albeit imperfect assessment of MPR. In other words, most patients do not continue to refill medications they are not taking, especially if their effort and funds are required. Medications that are 'automatically' mailed to the patient's home provide less useful information with regard to the MPR as a proxy for adherence. Reducing outof-pocket costs and prescribing single-pill combinations can improve adherence and control. Addressing side effects and engaging uncontrolled aTRH patients in $\mathrm{BP}$ self-monitoring are proven strategies for improving adherence and control. ${ }^{2,8}$ Self-monitoring as a strategy to improve hypertension control is more effective when linked with relaying BP information to their clinician's office and when the patient receives advice from the clinician or staff to improve blood presure management between scheduled office visits.

Adherence with a healthy lifestyle is also important in uncontrolled and TRH. The benefits of sodium restriction ${ }^{9}$ and avoiding excess alcohol and drugs, such as amphetamines and cocaine are important. ${ }^{2}$ Most obese patients will have a fall in BP with weight loss. ${ }^{2}$ The dietary approaches to stop hypertension (DASH) eating plan lowers BP without weight loss. ${ }^{10}$ Moreover, DASH appears even more effective in lowering BP and reducing other cardiovascular risk factors when it is the nutritional component of a weight loss plan. ${ }^{11}$

\section{Secondary Hypertension}

Once common contributors to pseudoresistant hypertension are determined to be unlikely explanations for aTRH, further evaluation is important. Suspected secondary causes of hypertension can be assessed in most office settings. ${ }^{1}$ Patients with resistant hypertension are often obese and volume expanded, which contributes 
to sleep apnea. ${ }^{12}$ Better control of volume, e.g. adding spironolactone, can improve BP and sleep apnea. ${ }^{13}$ While treatment of sleep apnea with measures, such as continuous positive airways pressure (CPAP), can improve hypertension control, the impact on $\mathrm{BP}$ is often unimpressive, even in patients that adhere with and otherwise benefit from this therapy. ${ }^{14}$

Primary aldosteronism is the most common secondary hypertension, ${ }^{1}$ but not all patients are hypokalemic. Plasma renin activity is suppressed together with excess aldosterone production. ${ }^{15}$ While an aldo/renin ratio $>20-30: 1$ is a screening test for primary aldosteronism, patients with low-renin hypertension can satisfy ratio criteria without having aldosterone excess. Marked hypokalemia can reduce aldosterone production and should be corrected prior to evaluation. Preferably, patients are not on medications impacting the renin-angiotensinaldosterone axis for several weeks prior to the assessment for primary aldosteronism. Practically, a disproportionate share of patients with TRH has suppressed plasma renin activity on treatment, although patients should not be taking aldosterone antagonists or epithelial sodium channel antagonists, e.g. amiloride, when assessing primary aldosteronism. If low plasma renin occurs with excess aldosterone in a 24-hour urine, the diagnosis is confirmed by failure to suppress aldosterone with saline infusion or a high salt diet. The majority of patients with primary aldosteronism have bilateral adrenal hyperplasia, rather than aldosterone-producing adenoma. If a unilateral adrenal lesion is identified on an imaging procedure, such as computerized tomographic scanning, confirmation of excess aldosterone from that gland is important prior to surgery as incidental, inactive adrenal lesions are relatively common. Patients with aldosterone-producing adenoma and bilateral adrenal hyperplasia often respond to aldosterone antagonists with other agents, e.g. thiazide diuretics and calcium antagonists.

Other secondary forms of hypertension including renal artery stenosis, chronic kidney disease, sleep apnea, pheochromocytoma, primary hyperparathyroidism, Cushing's syndrome and disease, aortic coarctation, hypo- and hyperthyroidism. ${ }^{1}$ Screening tests for secondary hypertension are provided in Table 2.1,15,16 Not unexpectedly, most screening tests listed, while useful, are associated with false negative (imperfect sensitivity) and false positive (imperfect specificity). When the 'pretest' likelihood of a specific secondary form of hypertension is high, then confirmatory testing is appropriate in the event a screening test was used and negative.

\section{Interfering Substances and Medications}

Various over-the-counter and prescribed medications, e.g. oral contraceptives, nonsteroidal anti-inflammatory
Table 2: Screening tests for selected causes of treatment resistant hypertension

\begin{tabular}{ll}
\hline $\begin{array}{l}\text { Suspected secondary } \\
\text { hypertension }\end{array}$ & Screening test \\
\hline Renal artery stenosis & $\begin{array}{l}\text { Duplex Doppler ultrasonograp } \\
\text { (can be technically difficult), } \\
\text { computed tomographic angio- } \\
\text { graphy (CTA), magnetic res } \\
\text { nance angiography (MRA) }\end{array}$ \\
$\begin{array}{l}\text { Hypothyroidism, } \\
\text { hyperthyroidism }\end{array}$ & $\begin{array}{l}\text { 24-hour urine fractionated } \\
\text { metanephrine and } \\
\text { normetanephrine } \\
\text { Overnight dexamethasone }\end{array}$ \\
Cushing's syndrome/disease & $\begin{array}{l}\text { Overnom } \\
\text { suppression test } \\
\text { Ca }\end{array}$ \\
Primary hyperparathyroidism & $\begin{array}{l}\text { History, polysomnography } \\
\text { (sleep study) }\end{array}$ \\
Sleep apnea & consult up to date for additional
\end{tabular}

Readers are encouraged to consult up to date for additional specific information on screening test for the various causes on secondary hypertension above (Available at: http://www.uptodate. com/contents/search)

drugs, glucocorticoids, and other immunosuppressive agents calcineurin inhibitors (cyclosporine, tacrolimus), erythropoietin, and agents interfering with vascular endothelial growth factor signaling can raise BP. ${ }^{1}$ When possible, these agents should be minimized or discontinued.

\section{Additions and Changes to the Pharmacological Regimen}

Adding a low-dose aldosterone antagonist, e.g. 12.5 to $50 \mathrm{mg}$ spironolactone, lowers BP in many TRH patients. 1,15,16 If patients are not ideal candidates for spironolactone, e.g. baseline serum $\mathrm{K}^{+}>4.5$ or eGFR $<45 \mathrm{ml} / 1.7 \mathrm{~m}^{2} / \mathrm{min}^{17}$ then changing from hydrochlorothiazide (HCTZ) to chlorthalidone can lower BP. Chlorthalidone is more likely than HCTZ to be effective with eGFR 30-44 (stage 3B CKD). Patients with eGFR $<30 \mathrm{ml} / 1.7 \mathrm{~m}^{2} / \mathrm{min}$, may require a loop diuretic, e.g. torsemide to improve volume control and lower BP. Patients with eGFR $<15$ may require dialysis for volume and BP control.

In patients with progesterone and antiandrogenic effects of spironolactone, eplerenone is an aldosterone antagonist devoid of sex-steroid effects. Since aldosterone raises BP in significant part by increasing the number and activity of epithelial sodium channels (ENaCs), amiloride, which blocks ENaCs, is also effective therapy for primary aldosteronism, especially at higher doses of 10 to $40 \mathrm{mg}$ daily. Monitoring for hyperkalemia is important with both aldosterone antagonists and epithelial sodium channel antagonists. Aldosterone antagonists and ENaC inhibitors have a low-risk when started at modest doses in individuals with eGFR $>45$ and serum potassium $<4.6 \mathrm{mmol} / \mathrm{l}$. While these agents can be used cautiously 
when essential in individuals with lower eGFR or higher serum potassium, beginning at very low doses and frequent monitoring (e.g. $\leq 7$ days), for adverse effects is warranted, especially when initiating treatment or increasing doses.

For patients with neurogenic hypertension, alpha and/or beta-receptor blockade or central sympatholytics, e.g. guanfacine, can lower BP. Hemodynamic, and reninguided therapy can identify effective medications. ${ }^{18,19}$ With renin-guided therapy, evidence suggests that lowrenin patients have a better $\mathrm{BP}$ response to diuretics, aldosterone antagonists, $\alpha_{1}$-adrenoceptor blockers, and calcium antagonists. Conversely, high-renin patients can have more robust $\mathrm{BP}$ responses to angiotensin converting enzyme inhibitors or angiotensin receptor, $\beta$-adrenoceptor, and renin blockers. Middle renin patients can respond well to both groups of medications with preference given to the group not prescribed. For example, in a patient with medium renin values on a beta-blocker and angiotensin receptor blocker, a diuretic or nondihydropyridine calcium antagonist would be preferred and vice versa. In the absence of compelling indicating for both the beta-blocker and angiotensin receptor blocker, e.g. heart failure or previous myocardial infarction, then the betaor angiotensin receptor blocker could potentially be withdrawn with maintenance of hypertension control.

\section{Prognosis in Patients with Controlled and Uncontrolled TRH}

Hypertension guidelines generally recommend adding and uptitrating antihypertensive medications until goal $\mathrm{BP}$ is achieved. ${ }^{20-23}$ The implied assumption is that the benefits of treating hypertension are mainly related to $\mathrm{BP}$ lowering irrespective of the number of antihypertensive medications required to achieve $\mathrm{BP}$ reduction. However, one study reported that patients with aTRH, that included both controlled and uncontrolled aTRH, have worse outcomes than uncontrolled hypertensive patients without aTRH. ${ }^{24}$ The treat to new targets cholesterol study noted that patients with both controlled and uncontrolled aTRH had a similar and greater risk for cardiovascular events than uncontrolled patients without aTRH. ${ }^{25}$ Two other reports indicate that patients with aTRH, including controlled aTRH, are at greater risk for one or more CVD events or death than patients without aTRH. ${ }^{26,27}$ Moreover, Valsartan antihypertensive long-term use evaluation (VALUE) trial data indicated that patients controlled on a single medication had significantly fewer cardiovascular events than patients controlled on combination therapy, even after adjusting for baseline BP and history of cardiovascular disease. ${ }^{28}$
In fact, cardiovascular outcomes were not significantly different between controlled and uncontrolled patients on combination therapy.

Thus, in patients uncontrolled on three or more are controlled on four or more medications, it is important for clinicians to recognize that these individuals likely remain at higher risk for cardiovascular events than patients prescribed fewer medications. To mitigate excess risk, clinicians are encouraged to optimize control of other cardiovascular risk factors with special attention to cholesterol in statin eligible patients. ${ }^{29}$ In addition, even moderate dose statin, e.g. $10 \mathrm{mg}$ atorvastatin in the Anglo-Scandinavian Outcomes Trial (ASCOT) was associated with a lower likelihood of treatment resistant hypertension. ${ }^{30}$ This is consistent with other evidence suggesting a mild BP lowering effect of statins, which appears to be greater in patients with hypertension than those with nonhypertensive BP values. ${ }^{31}$ Clinicians are also encouraged to use antihypertensive medications with compelling indications ${ }^{32}$ in patients with diabetes, chronic kidney disease, and cardiovascular disease, all of which heighten future risk for incident cardiovascular events.

In summary, aTRH is a common condition. Using the pragmatic approach outlined, clinicians can identify and address pseudoresistance, screen for secondary hypertension and initiate changes to lifestyle and pharmacotherapy to improve BP control. It is likely that $\geq 80 \%$ of aTRH patients can be successfully managed in primary care. For complex cases of secondary hypertension and truly refractory hypertension, referral to a hypertension specialist is warranted for further management and consideration of device-based therapies.

\section{REFERENCES}

1. Calhoun DA, Jones D, Textor S, et al. American Heart Association Professional Education Committee. Resistant hypertension: diagnosis, evaluation, and treatment: a scientific Statement from the American Heart Association Professional Education Committee of the Council for High Blood Pressure Research. Hypertension 2008 Jun;51(6): 1403-1419.

2. Egan BM, Zhao Y, Axon RN, Brzezinski WA, Ferdinand KD. Uncontrolled and apparent treatment resistant hypertension in the US 1988-2008. Circulation 2011 Aug 30;124(9):1046-1058.

3. Modan M, Halkin H, Almog S, Lusky A, Eshkol A, Shefi M, Shitrit A, Fuchs Z. Hyperinsulinemia: a link between hypertension, obesity and glucose intolerance. J Clin Invest 1985 Mar;75(3):809-817.

4. Egan BM, Zhao Y, Li J, Brzezinski WA, Todoran TM, Brook RD, Calhoun DA. Prevalence of optimal treatment regimens in patients with apparent treatment resistant hypertension in a community-based practice network. Hypertension 2013 Oct;62(4):691-697. 
5. de la Sierra A, Segura J, Banegas JR, Gorostidi M, de la Cruz JJ, Armario P, Oliveras A, Ruilope LM. Clinical features of 8295 patients with resistant hypertension classified on the basis of ambulatory blood pressure monitoring. Hypertension 2011 May;57(5):898-902.

6. Salles GF, Cardoso CR, Muxfeldt ES. Prognostic influence of office and ambulatory blood pressures in resistant hypertension. Arch Intern Med 2008 Nov 24;168(21):2340-2346.

7. Pierdomenico SD, Lapenna D, Bucci A, Di Tommaso R, Di Mascio R, Manente BM, Caldarella MP, Neri M, Cuccurullo F, Mezzetti A. Cardiovascular outcome in treated hypertensive patients with responder, masked, false resistant, and true resistant hypertension. Am J Hypertension 2005 Nov; 18(11):1422-1428.

8. Siu AL. Screening for high blood pressure in adults: US Preventive Services Task Force Recommendation Statement. Ann Intern Med 2015 Nov 17;163(10). DOI: 10.7326/P15-9036. Epub 2015 Oct 13.

9. Mancia G, Facchetti R, Bombelli M, Grassi G, Sega R. Longterm risk of mortality associated with selective and combined elevation in office, home, and ambulatory blood pressure. Hypertension 2006 May;47(5):846-853.

10. Myers MG, Kaczorowski J, Dawes M, Godwin M. Automated office blood pressure measurement in primary care. Can Fam Physician 2014 Feb;60(2):127-132.

11. Azadbakht L, Azizi T, Mirmiran P, Azizi F, Esmaillzadeh A. Beneficial effects of a dietary approaches to stop hypertension eating plan on features of the metabolic syndrome. Diabetes Care 2005 Dec;28(12):2823-2831.

12. Friedman O, Bradley TD, Chan CT, Parkes R, Logan AG. Relationship between overnight rostral fluid shift and obstructive sleep apnea in drug-resistant hypertension. Hypertension 2010 Dec;56(6):1077-1082.

13. Gaddam K, Pimenta E, Thomas SJ, Cofield SS, Oparil S, Harding SM, Calhoun DA. Spironolactone reduces severity of obstructive sleep apnea in patients with resistant hypertension: a preliminary report. J Hum Hypertens 2010 Aug;24(8):532-537. DOI: 10.1038/jhh.2009.96. Epub 2009 Dec 17.

14. Schien AS, Kerkoff AC, Coronel CC, Plentz RD, Sbruzzi G. Continuous positive airway pressure reduces blood pressure in patients with obstructive sleep apnea: a systematic review and meta-analysis with 1000 patients. J Hypertens 2014 Sep; 32(9):1762-1773.

15. Mattsson C, Young WF Jr. Primary aldosteronism: diagnostic and treatment strategies. Nat Clin Pract Nephrol 2006 Apr; 2(4):198-208.

16. Kaplan NM, Calhoun DA. Treatment resistant hypertension. UpToDate. Available at: (http://www. uptodate.com/ contents/treatment-of-resistant-hypertension. Accessed on: 24 Aug 2015.

17. Khosla N, Kalaitzidis R, Bakris GL. Predictors of hyperkalemia risk following hypertension control with aldosterone blockade. Am J Nephrol 2009;30(5):418-424.

18. Laragh J. Laragh's lessons in pathophysiology and clinical pearls for treating hypertension. Lesson XVI: How to choose the correct drug for each hypertensive patient using a plasma renin-based method and the volume-vasoconstriction analysis. Am J Hypertens 2001;14(6):491-503.

19. Egan BM, Basile JN, Rehman SU, et al. Renin-guided algorithm matches clinical hypertension specialist care in uncontrolled hypertension: a randomized-controlled clinical trial. Am J Hypertens 2009 Jul;22(7):792-801.
20. Dasgupta K, Quinn RR, Zarnke KB, Rabi DM, Ravani P, Daskalopoulou SS, et al. Canadian Hypertension Education Program. The 2014 Canadian Hypertension Education Program recommendations for blood pressure measurement, diagnosis, assessment of risk, prevention, and treatment of hypertension. Can J Cardiol 2014 May;30(5):485-501.

21. Dolan E, Stanton A, Thija L, Atkins N, McClory S, Hond ED, McCormack P, Saessen JA, O'Brien E. Superiority of ambulatory over clinic blood pressure measurement in predicting mortality: the dublin outcome study. Hypertension 2005 Jul;46(1):156-161.

22. Ho PM, Bryson CL, Rumsfeld JS. Medication adherence: its importance in cardiovascular outcomes. Circulation 2009 Jun 16;119(23):3028-3035.

23. Bosworth HB, Powers BJ, Olsen MK, McCant F, Grubber J, Smith V, Gentry PW, Rose C, Van Houtven C, Wang V, et al. Home blood pressure management and improved blood pressure control: results from a randomized controlled trial. Arch Intern Med 2011 Jul 11;171(13):1173-1180.

24. Daugherty SL, Power JD, Magid DJ, Tavel HM, Oasoudi FA, Margolis KL, et al. Incidence and prognosis of resistant hypertension in hypertensive patients. Circulation $2012 \mathrm{Apr}$ 3;125(13):1635-1642.

25. Bangalore S, Fayyad R, Laskey R, DeMicco DA, Deedwania $\mathrm{P}$, Kostis JB, Messerli FH. Treating to New Targets Steering Committee and Investigators. Prevalence, predictors and outcomes in treatment-resistant hypertension in patients with coronary disease. Am J Med 2014 Jan;127(1):71-81.

26. Munter P, Davis BR, Cushman WC, Bangalore S, Calhoun DA, Pressel SL, Black HR, Kostis JB, Probstfield J, Whelton PK, et al. Treatment resistant hypertension and the incidence of cardiovascular disease and end-stage renal disease: results from the antihypertensive and lipid-lowering treatment to prevent heart attack (ALLHAT). Hypertension 2014 Nov;64(5):1012-1021.

27. Irvin MR, Booth JN III, Shimbo D, Lackland DT, Oparil S, Howard G, Safford MM, Muntner P, Calhoun DA. Apparent treatment-resistant hypertension and risk for stroke, coronary heart disease, and all-cause mortality. J Am Soc Hypertens 2014 Jun;8(6):405-413.

28. Weber MA, Julius S, Kjedlsen SE, Jia Y, Brunner HR, Zappe $\mathrm{DH}$, et al. Cardiovascular outcomes in hypertensive patients comparing single-agent therapy with combination therapy. J Hypertens 2012 Nov;30(11):2213-2222.

29. Egan BM, Li J, Qanungo S, Wolfman TE. Blood pressure and cholesterol control in hypertensive hypercholesterolemic patients: a report from NHANES 1988-2010. Circulation 2013 Jul 2;128(1):29-41.

30. Gupta AK, Nasothimiou EG, Chang CL, Sever PS, Dahlöf $B$, Poulter NR. ASCOT investigators. Baseline predictors of resistant hypertension in the Anglo-Scandinavian Cardiac Outcome Trial (ASCOT): a risk score to identify those at high-risk. J Hypertens 2011 Oct;29(10):2004-2013.

31. Briasoulis A, Agarwal V, Valachis A, Messerli FH. Antihypertensive effects of statins: a meta-analysis of prospective controlled studies. J Clin Hypertens (Greenwich) 2013 May; 15(5):310-320.

32. Chobanian AV, Bakris GL, Black HR, Cushman WC, Green LA, Izzo JL Jr, Jones DW, Materson BJ, Oparil S, Wright JT Jr, et al. Seventh report of the Joint National Committee on Prevention. Detection, evaluation, and treatment of high blood pressure. Hypertension 2003 Dec;42(6):1206-1252. 